\title{
Associations between thyroid hormones and serum metabolite profiles in an euthyroid population
}

\author{
Carolin Jourdan · Jakob Linseisen • Christa Meisinger • Ann-Kristin Petersen • \\ Christian Gieger - Rajesh Rawal - Thomas Illig • Margit Heier • Annette Peters • \\ Henri Wallaschofski • Matthias Nauck · Gabi Kastenmüller • Karsten Suhre • \\ Cornelia Prehn • Jerzy Adamski - Wolfgang Koenig • Michael Roden • \\ H-Erich Wichmann · Henry Völzke
}

Received: 5 March 2013/Accepted: 28 June 2013/Published online: 18 July 2013

(C) Springer Science+Business Media New York 2013

\begin{abstract}
The aim was to characterise associations between circulating thyroid hormones-free thyroxine (FT4) and thyrotropin (TSH) — and the metabolite profiles in serum samples from participants of the German populationbased KORA F4 study. Analyses were based on the metabolite profile of 1463 euthyroid subjects. In serum samples, obtained after overnight fasting $(\geq 8), 151$ different metabolites were quantified in a targeted approach including amino acids, acylcarnitines (ACs), and phosphatidylcholines (PCs). Associations between metabolites and thyroid hormone concentrations were analysed using adjusted linear regression models. To draw conclusions on thyroid hormone related pathways, intra-class metabolite ratios were
\end{abstract}

Electronic supplementary material The online version of this article (doi:10.1007/s11306-013-0563-4) contains supplementary material, which is available to authorized users.

C. Jourdan · J. Linseisen ( $\square) \cdot$ C. Meisinger · H.-E. Wichmann Institute of Epidemiology I, Helmholtz Zentrum München, German Research Centre for Environmental Health (HMGU), Ingolstädter Landstraße 1, 85746 Neuherberg, Germany

e-mail: j.linseisen@helmholtz-muenchen.de

C. Meisinger $\cdot$ M. Heier $\cdot$ A. Peters

Institute of Epidemiology II, Helmholtz Zentrum München,

Neuherberg, Germany

A.-K. Petersen · C. Gieger - R. Rawal

Institute of Genetic Epidemiology, Helmholtz Zentrum

München, Neuherberg, Germany

T. Illig · A. Peters

Research Unit of Molecular Epidemiology, Helmholtz Zentrum

München, Neuherberg, Germany

T. Illig

Hannover Unified Biobank, Hannover Medical School,

Hannover, Germany additionally explored. We discovered 154 significant associations (Bonferroni $p<1.75 \times 10^{-04}$ ) between FT4 and various metabolites and metabolite ratios belonging to $\mathrm{AC}$ and PC groups. Significant associations with TSH were lacking. High FT4 levels were associated with increased concentrations of many ACs and various sums of ACs of different chain length, and the ratio of $\mathrm{C} 2$ by $\mathrm{C} 0$. The inverse associations observed between FT4 and many serum PCs reflected the general decrease in PC concentrations. Similar results were found in subgroup analyses, e.g., in weightstable subjects or in obese subjects. Further, results were independent of different parameters for liver or kidney function, or inflammation, which supports the notion of an independent FT4 effect. In fasting euthyroid adults, higher serum FT4 levels are associated with increased serum AC concentrations and an increased ratio of $\mathrm{C} 2$ by $\mathrm{C} 0$ which is

H. Wallaschofski · M. Nauck

Institute of Clinical Chemistry and Laboratory Medicine, Ernst-

Moritz-Arndt-University Greifswald, Greifswald, Germany

G. Kastenmüller · K. Suhre

Institute of Bioinformatics and Systems Biology, Helmholtz

Zentrum München, Neuherberg, Germany

K. Suhre

Department of Physiology and Biophysics, Weill Cornell

Medical College, Education City, Doha, Qatar

C. Prehn · J. Adamski

Institute of Experimental Genetics, Genome Analysis Center,

Helmholtz Zentrum München, Neuherberg, Germany

J. Adamski

Institute of Experimental Genetics, Life and Food Science

Center Weihenstephan, Technische Universität München,

Freising-Weihenstephan, Germany 
indicative of an overall enhanced fatty acyl mitochondrial transport and $\beta$-oxidation of fatty acids.

Keywords Targeted metabolomics - Serum metabolites . Free thyroxine - Thyrotropin . Thyroid hormones . Epidemiology

\section{Introduction}

Thyroid hormones such as thyroxine (T4) and triiodothyronine (T3) are responsible for the proper function of nearly all organ systems (Brix et al. 2011). They do not only modulate the metabolism of carbohydrates and lipids, influence protein synthesis, affect the cellular reaction to catecholamines in almost every tissue; they are also unique in their ability to influence all over energy expenditure, e.g., via the regulation of thermogenesis (Kim 2008; Panicker et al. 2010; Moreno et al. 2008). The release of T4 and T3 by the thyroid gland is controlled by thyroid stimulating hormone (thyrotropin; TSH) which is synthesized by the anterior pituitary gland in response to TSHreleasing hormone, which in turn is secreted by the hypothalamus. T4 is the quantitatively main product of the thyroid gland (Yen 2001). Almost all of T3 and T4 in the circulation are protein-bound (Boelaert and Franklyn 2005); only about 0.03 and $0.3 \%$ of total serum T4 and T3, respectively, are free or unbound (Yen 2001). The free thyroid hormones (FT4 and FT3) actively enter target cells and trigger biological reactions (Yen 2001). Perturbations of thyroid hormone release and functioning belong to the most common endocrine disorders and are connected to major health problems (Brix et al. 2011). A prolonged imbalance of thyroid hormones leads to diseases, such as hyperthyroidism or hypothyroidism, and has been linked to

\footnotetext{
W. Koenig

Department of Internal Medicine II-Cardiology, University of Ulm, Medical Center, Ulm, Germany

M. Roden

Institute for Clinical Diabetology, German Diabetes Center, Düsseldorf, Germany

H.-E. Wichmann

Institute of Medical Informatics, Biometry and Epidemiology, Chair of Epidemiology, Ludwig-Maximilians-Universität

München, Neuherberg, Germany

H.-E. Wichmann

Klinikum Großhadern, Munich, Germany

H. Völzke

Institute for Community Medicine, Ernst-Moritz-Arndt-

University Greifswald, Greifswald, Germany
}

an increased risk for cardiovascular morbidity and mortality (Brix et al. 2011; Boelaert and Franklyn 2005; Biondi 2012b). The circulating concentrations of TSH, FT4 and FT3 have a strong heritable component and are supposed to be controlled by multiple genes (Panicker et al. 2010). However, in the case of FT4 these genes are still unknown.

In the present work we investigate the association between FT4 concentrations and serum metabolite profiles. The concept of targeted metabolomics is the quantification of a defined set of metabolites in a body fluid, representing an image of the current metabolic state of the organism (Altmaier et al. 2009). It has previously been shown that this method has the power to identify perturbations of the body's metabolic homeostasis and allows for the identification of and access to biomarkers of metabolic pathways that are impacted, for example, by diseases (Suhre et al. 2010; Altmaier et al. 2008; Vinayavekhin and Saghatelian 2009; Watson 2006; Dumas et al. 2007; Assfalg et al. 2008).

The focus of the present study was on euthyroid participants, i.e., participants with FT3, FT4, and TSH levels in the normal range, no history of known thyroid diseases, and no intake of thyroid-specific medication. We hypothesised that within the normal ranges of FT4 and TSH an effect on substrate oxidation exists, and that the activity of involved enzymes is reflected in serum metabolite concentrations. Therefore, we took a targeted quantitative metabolomics approach to identify FT4 and TSH triggered pathways and differences in varying population strata by, e.g., gender, obesity status, age, or physical activity.

\section{Materials and methods}

\subsection{Ethics statement}

The study was approved by the ethics committee of the Bavarian Medical Association. Written informed consent was obtained from each participant in accordance with institutional requirements and the Declaration of Helsinki Principles.

\subsection{Study design and population}

The present analyses are based on subsamples of the metabolically characterised subjects who participated in the KORA F4 study, the seven year follow-up of the KORA S4 study. KORA (Cooperative Health Research in the Region of Augsburg) is a research platform performing population-based surveys and subsequent follow-ups in the region of Augsburg in Southern Germany and has been described in detail elsewhere (Holle et al. 2005). The 
metabolic profiles of 3044 participants in KORA F4, aging 31-82 years, were determined. For the present analyses only euthyroid participants were included. A person was defined as euthyroid if their FT3, FT4 and TSH hormone levels were in the ranges of $3.3-6.1 \mathrm{pmol} / \mathrm{L}, 9.8$ $18.8 \mathrm{pmol} / \mathrm{L}$ and $0.3-4.0 \mathrm{mIU} / \mathrm{L}$ according to the assay manuals and Brabant et al. (2006) as well as Surks et al. (2004), respectively, their thyroid peroxidase antibody level did not exceed $200 \mathrm{IU} / \mathrm{mL}$ (Demers and Spencer 2003; Knudsen et al. 1999) and there was no known history of thyroid disease or thyroid medication use in accordance with the anatomical therapeutic chemical code for thyroid therapy (H03). Further, subjects with a known history of myocardial infarction, stroke, diabetes or cancer were excluded as well as subjects taking ACE inhibitors or antilipidemic drugs, and women being pregnant at the time of blood collection. The selection process of the study participants is displayed in Fig. 1. Participants were excluded for several reasons if applicable. For a subanalysis, data on the participants' body composition was used. However, the bioelectrical impedance analysis measurements were only conducted in the KORA S4 study which was seven years prior to the KORA F4 examinations. To account for this, only weight-stable participants of the KORA F4 population were included in this subanalysis. Persons were defined as weight-stable if their weight gain or loss did not exceed more than $0.5 \%$ per year between measurements in KORA S4 and F4 (Nimptsch et al. 2010). This resulted in a sample size of $n=1463$ for KORA F4 and of $n=616$ for the weight-stable subgroup in KORA F4.

\subsection{Blood sampling}

Blood samples were drawn between 8:00 and 10:30 a.m. after a period of overnight fasting (minimum of $8 \mathrm{~h}$ ). S-Monovette ${ }^{\circledR}$ serum gel tubes (SARSTEDT AG \& Co., Nümbrecht, Germany) were used. After blood was drawn, tubes were inverted twice and then allowed to rest for $30 \mathrm{~min}$ at room temperature $\left(18-25^{\circ} \mathrm{C}\right)$ to achieve total coagulation. Tubes were centrifuged for $10 \mathrm{~min}$ at 2,660 g at $15{ }^{\circ} \mathrm{C}$ and the obtained serum was divided into aliquots, chilled for a maximum of $6 \mathrm{~h}$ at $4{ }^{\circ} \mathrm{C}$ before being stored at $-80{ }^{\circ} \mathrm{C}$ until analysis.

\subsection{Blood analyses}

Concentrations of TSH, FT3, and FT4 were analysed by immunochemiluminescent procedures (Dimension Vista System, Siemens, Germany). The functional sensitivity of the TSH assay was $0.005 \mathrm{mIU} / \mathrm{L}$; the TSH, FT3, and FT4 working ranges were $0.005-100 \mathrm{mIU} / \mathrm{L}, 0.77-46.1 \mathrm{pmol} / \mathrm{L}$, and $1.3-103 \mathrm{pmol} / \mathrm{L}$, respectively. The (low or high) interassay coefficients of variations were 2.0 or $2.2 \%$ for TSH, and 1.7 or $4.1 \%$ for FT4. All assays were performed according to the manufacturer's recommendations. However, the applied analytic method for FT3 did not fulfil the strict quality control criteria in the end (Thienpont et al. 2010). Thus, data on FT3 was only used for the definition of euthyroid subjects but not for analyses. The thyroid peroxidase antibodies were determined by an enzyme immunoassay (VARELISA, Elias Medizintechnik
Fig. 1 Flowchart of the selection process of the study participants for the present analyses. (Participants were excluded for multiple reasons.)

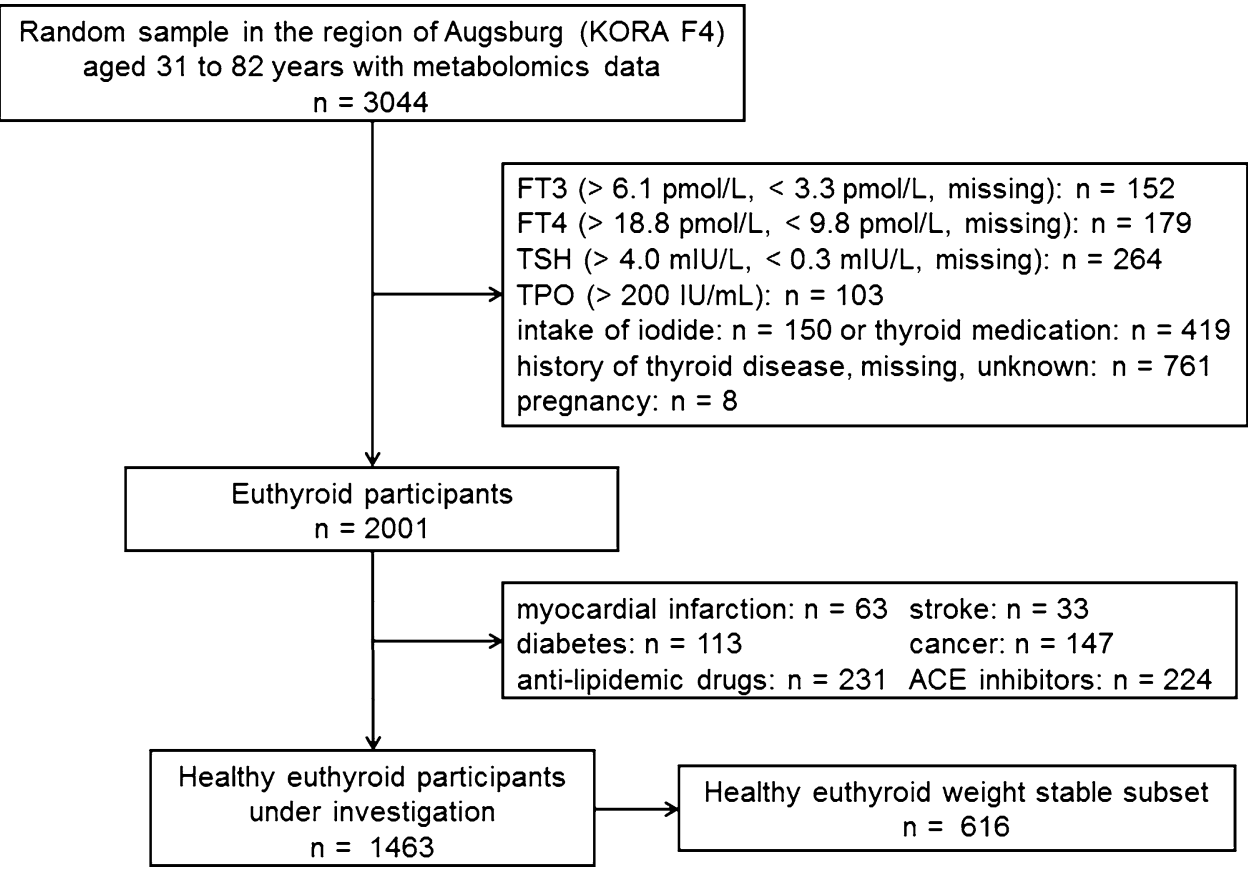


$\mathrm{GmbH}$, Freiburg, Germany) with a functional sensitivity of $1 \mathrm{IU} / \mathrm{mL}$.

The applied analyses methods to determine glutamate pyruvate transaminase, glutamic oxaloacetic transaminase, gamma-glutamyl transpeptidase, C-reactive protein (Ruckert et al. 2011), cystatin C (Goek et al. 2012), and creatinine (Seissler et al. 2012), concentrations have been described earlier. White blood cell count was determined from fresh venous EDTA blood using impedance measurements (Coulter ${ }^{\circledR}$ LH 780, Beckman Coulter Inc., USA).

\subsection{Serum metabolite quantification}

Metabolic characterisation of the KORA F4 serum samples was done in three batches of approximately 1000 samples at three different time points with a recalibration of the equipment in between.

The targeted metabolomics approach for KORA F4 was based on ESI-MS/MS measurements by AbsoluteID $Q^{\mathrm{TM}}$ p150 kit (BIOCRATES, Life Sciences AG, Innsbruck, Austria). The assay allows the simultaneous quantification of 163 metabolites out of $10 \mu \mathrm{l}$ serum. The Absolute $I D Q^{\mathrm{TM}} \mathrm{p} 150$ kit has been described in detail previously (Illig et al. 2010; Römisch-Margl et al. 2011) Sample handling was performed by a Hamilton Micro Lab Star robot (Hamilton Bonaduz AG, Bonaduz, Switzerland) and a Ultravap nitrogen evaporator (Porvair, Sciences, Leatherhead, United Kingdom). Mass spectrometric (MS) analyses were done on a 4000 QTRAP mass spectrometer (AB Sciex) coupled to Promincence HPLC (Shimadzu) apparatus equipped with a SIL-20AC autosampler and controlled by the software Analyst 1.4. Data evaluation for quantification of metabolite concentrations and quality assessment was performed with the $\operatorname{Met} I Q^{\mathrm{TM}}$ software package, which is an integral part of the Absolute $I D Q^{\mathrm{TM}}$ kit. Internal standards serve as reference for the calculation of metabolite concentrations. The methods of the AbsoluteIDQ $Q^{\mathrm{TM}}$ p150 kits has been proven to be in conformance with the FDA guideline "Guidance for Industry-Bioanalytical Method Validation, May 2001" (U.S. Department of Health and Human Services et al. May 2001), which implies proof of reproducibility within a given error range. Measurements were performed as described in the manufacturer manuals. Concentrations are reported in $\mu \mathrm{mol} / \mathrm{L}$.

\subsection{Metabolite spectrum}

In total, 163 different metabolites were detected, including 14 amino acids (13 proteinogenic + ornithine), hexose (sum of hexoses-about 90-95\% glucose), free carnitine and 40 acylcarnitines (Cx:y), 15 sphingomyelins (SMx:y), 77 phosphatidylcholines (PCs, aa = diacyl, ae = acyl-alkyl) and 15 lyso-phosphatidylcholines (lysoPCs). The lipid side chain composition is abbreviated as $\mathrm{C} x$ : $y$, with $x$ denoting the number of carbons in the side chain and $y$ denoting the number of double-bonds. Full biochemical names are provided in the Supplementary Table S1.

\subsection{Quality control of the metabolomics dataset}

The quality control of the metabolomics dataset of KORA F4 was done in a two-step procedure. First, the quality of all metabolites was assessed using a reference blood which was measured five times on ten plates. With this data, a coefficient of variation was calculated for every metabolite and plate. All metabolites having a mean coefficient of variation over all ten plates bigger than $25 \%$ were removed from the dataset (11 in total). One further metabolite was excluded as the number of missing values exceeded $5 \%$. In the second step, the dataset was controlled for outliers. A subject's metabolite concentration was defined as an outlier if the concentration was greater or less than the mean \pm five standard deviations of the particular metabolite over the whole population. All subjects having more than three independent outlying metabolite concentrations were excluded from the dataset. An outlier was defined as independent if the correlation with all other outliers was less than $70 \%$. If there were three or less independent outliers, only the data points were removed. All missing values $(<0.09 \%)$ were imputed with the $\mathrm{R}$ package "mice" (van Buuren and Groothuis-Oudshoorn 2011) which uses a linear regression approach (predictive mean matching; vector size $=151$ ). This left us with 151 metabolites.

\subsection{Anthropometry, physical activity and body composition assessment}

Height and weight were measured to the nearest $0.1 \mathrm{~cm}$ and $0.1 \mathrm{~kg}$, respectively. The body mass index (BMI) was calculated as weight in $\mathrm{kg} /(\text { height in } \mathrm{m})^{2}$ and according to the world health organisation (WHO) obesity was defined as having a $\mathrm{BMI} \geq 30 \mathrm{~kg} / \mathrm{m}^{2}$ (WHO 2000). Waist circumference was measured to the closest $0.1 \mathrm{~cm}$ at the smallest position between the lower rip and the upper margin of the iliac crest. Hip size was determined exactly to $0.1 \mathrm{~cm}$ as the widest circumference measured between the upper margin of the iliac crest and the crotch. Physical activity was assessed on a four-level graded scale by the amount of regular leisure time exercise per week during summer and wintertime. Based on those assessments, Meisinger et al. (2007) defined four levels of physical activity: (i) 'No sports activities in leisure time' - almost no sports activity or no activity in summer and in winter; (ii) 'Low level of sports activities in leisure time'-irregular exercise of $1 \mathrm{~h}$ 
per week at least in summer or winter; (iii) 'Moderate level of sports activity in leisure time'-regular exercise of $1 \mathrm{~h}$ per week at least in summer or winter; (iv) 'High level of sports activities in leisure time'-more than $2 \mathrm{~h}$ per week of regular exercise in summer and winter. Based on this variable, the physical activity variable which was used in the present analyses was created representing two levels: 'physically inactive' — category (i) or (ii), and 'physically active' - category (iii) or (iv). For the assessment of body composition in KORA S4, two bioelectrical impedance analysis measurements of resistance $(\mathrm{R})$, reactance $(\mathrm{Xc})$ and the phase angle $(\alpha)$ were taken between the dominant hand wrist and dorsum and the dominant foot angle and dorsum (placement of the electrodes) by means of a body impedance analyser (BIA 2000-S; Data Input GmbH, Frankfurt, Germany) while subjects were spreading their arms and legs and lying in a relaxed and supine position on a nonconductive surface with $50 \mathrm{kHz}$. Fat free mass was calculated by means of Kyle's equation (Kyle et al. 2001). In accordance to the BMI calculation, a fat free mass index (FFMI) (fat free mass in $\mathrm{kg} /(\text { height in } \mathrm{m})^{2}$ ) was determined.

\subsection{Statistical analyses}

The descriptive data is presented as mean and standard deviation for the continuous variables and as absolute quantities and percentages for the discrete parameters. Differences in hormone concentrations across different categories were tested by means of pairwise t-tests. Besides the absolute metabolite concentrations, all pairs of intrametabolite class ratios $(n=2188)$ were part of the metabolomics dataset. Associations between metabolite concentrations or metabolite ratios and FT4 or TSH hormone levels were assessed by means of linear models. First, metabolite variables were standardised with an inverse lognormal transformation to allow for comparison of the estimates derived from the linear regression models. Then, linear regression models were applied with the metabolite variables as dependent variables and the hormone concentrations as independent variable. The linear models were adjusted for age, sex, BMI, and batch. To control for multiple testing and with regard to the dependencies among the various metabolites and ratios, a $p$ value of $1.75 \times 10^{-4}$ was considered statistically significant at $\alpha=5 \%$. For an association with a metabolite ratio to be regarded as statistically significant, a further criterion (p-gain) had to be fulfilled next to a significant $p$ value. The p-gain is defined as the fold decrease in the $p$ value of the association for the pair of metabolites compared to the lowest of the two $p$ values for the single metabolites (Suhre et al. 2010). Thus, an association between a metabolite ratio (M1/M2) and FT4 or TSH is considered to be significant, if the $p$ value of this association is significant and the p-gain exceeds a certain value. To calculate the p-gain we first have to determine the minimum $p$ value of the associations between metabolite M1 and FT4 or TSH as well as metabolite M2 and FT4 or TSH. The p-gain is the quotient of this minimum to the $p$ value of the association between the metabolite ratio and FT4 or TSH. This p-gain had to exceed $2.86 \times 10^{+03}$. This cut-off is seen as Bonferroni-related correction in order to identify metabolite concentration pairs for which the strength of association improves considerably by using ratios (Suhre et al. 2010; Jourdan et al. 2012).

\subsubsection{Subanalyses}

In a subanalysis, the effect of FFMI on the association between FT4 and the metabolomics data was examined. Therefore, the weight-stable set was used and the applied linear models were adjusted for FFMI instead of BMI. Stratified analyses of the entire sample were also conducted by sex, obesity status $\left(\mathrm{BMI} \geq 30 \mathrm{~kg} / \mathrm{m}^{2}\right.$ versus $\mathrm{BMI}<$ $30 \mathrm{~kg} / \mathrm{m}^{2}$ ), age groups ( $\leq 60$ years versus $>60$ years), and physical activity as potential confounder or effect modifiers. The equality of the strata-specific regression coefficients was tested by means of an approximately normally distributed test-statistic as described by Paternoster et al. (Paternoster et al. 1998). Further, the influence of serum insulin, different liver, kidney, and inflammation parameters on the identified associations between the metabolites and FT4 were analysed by including an interaction term between FT4 and those parameters in the described main model.

All statistical analyses were performed with the $\mathrm{R}$ software version 2.12.0 [R Development Core Team, 2010, http://www.r-project.org].

\section{Results}

Table 1 summarises the characteristics of all study participants and the weight-stable subset. On average, men and women were $51 \pm 12$ years old and had a BMI of $27 \pm 4$ and $26 \pm 5 \mathrm{~kg} / \mathrm{m}^{2}$, respectively; $19-20 \%$ of men and women were classified as obese. Mean serum TSH and FT4 concentrations were $1.40 \pm 0.65 \mathrm{mIU} / \mathrm{L}$ and $13.45 \pm$ $1.67 \mathrm{pmol} / \mathrm{L}$ in men and $1.50 \pm 0.71 \mathrm{mIU} / \mathrm{L}$ and $13.55 \pm$ $1.62 \mathrm{pmol} / \mathrm{L}$ in women. Comparing serum FT4 and TSH hormone concentrations across different categories of age, sex, BMI, and physical activity for all KORA F4 participants and the two subsets, weight-stable and obese participants, we hardly detected any differences (Table 2). Applying ANOVA and pairwise t-tests, mean FT4 values of all KORA F4 participants for the age strata 61-70 and 71-80 years were significantly different to the other strata. 
Table 1 Characteristics (Mean $\pm \mathrm{SD}$; \% or $n$ absolute) of male and female subjects in the KORA F4 sample and the weight-stable subset

\begin{tabular}{|c|c|c|c|c|c|}
\hline & & \multicolumn{2}{|l|}{ All $(n=1463)$} & \multicolumn{2}{|c|}{ Weight-stable $^{\mathrm{a}}(n=616)$} \\
\hline & & Men $(n=792)$ & Women $(n=671)$ & $\operatorname{Men}(n=343)$ & Women $(n=273)$ \\
\hline Parameter & Unit & Mean \pm SD & Mean \pm SD & Mean \pm SD & Mean \pm SD \\
\hline Age & years & $50.94 \pm 12.08$ & $50.98 \pm 12.39$ & $53.34 \pm 12.06$ & $54.03 \pm 12.7$ \\
\hline Weight & $\mathrm{kg}$ & $85.31 \pm 13.02$ & $69.76 \pm 13.13$ & $83.29 \pm 11.50$ & $67.80 \pm 12.56$ \\
\hline Height & $\mathrm{cm}$ & $177.11 \pm 6.85$ & $162.93 \pm 6.68$ & $176.66 \pm 7.02$ & $162.48 \pm 6.44$ \\
\hline Body-mass-index & $\mathrm{kg} / \mathrm{m}^{2}$ & $27.19 \pm 3.76$ & $26.32 \pm 4.96$ & $26.68 \pm 3.31$ & $25.7 \pm 4.63$ \\
\hline Waist & $\mathrm{cm}$ & $97.19 \pm 11.35$ & $85.23 \pm 12.24$ & $95.89 \pm 10.09$ & $83.94 \pm 12.17$ \\
\hline Hip & $\mathrm{cm}$ & $105.61 \pm 8.06$ & $104.86 \pm 9.92$ & $104.31 \pm 6.26$ & $103.46 \pm 9.43$ \\
\hline Waist-to-hip ratio & & $0.92 \pm 0.06$ & $0.81 \pm 0.07$ & $0.92 \pm 0.06$ & $0.81 \pm 0.07$ \\
\hline FT4 & $\mathrm{pmol} / \mathrm{L}$ & $13.45 \pm 1.67$ & $13.55 \pm 1.62$ & $13.43 \pm 1.62$ & $13.57 \pm 1.61$ \\
\hline $\mathrm{TSH}$ & $\mathrm{mIU} / \mathrm{L}$ & $1.40 \pm 0.65$ & $1.50 \pm 0.71$ & $1.37 \pm 0.63$ & $1.47 \pm 0.69$ \\
\hline Fat free mass ${ }^{b}$ & $\mathrm{~kg}$ & $60.85 \pm 6.12$ & $43.28 \pm 5.21$ & $60.83 \pm 6.10$ & $43.13 \pm 5.19$ \\
\hline Body fat mass ${ }^{b}$ & $\%$ & $26.29 \pm 4.99$ & $35.62 \pm 5.72$ & $26.41 \pm 4.94$ & $35.54 \pm 5.62$ \\
\hline $\mathrm{FFMI}^{\mathrm{b}, \mathrm{c}}$ & $\mathrm{kg} / \mathrm{m}^{2}$ & $19.55 \pm 1.64$ & $16.39 \pm 1.84$ & $19.61 \pm 1.58$ & $16.40 \pm 1.78$ \\
\hline $\mathrm{BFMI}^{\mathrm{b}, \mathrm{d}}$ & $\mathrm{kg} / \mathrm{m}^{2}$ & $7.17 \pm 2.24$ & $9.44 \pm 3.24$ & $7.21 \pm 2.08$ & $9.39 \pm 3.14$ \\
\hline Insulin & $\mu \mathrm{IU} / \mathrm{ml}$ & $6.87 \pm 12.04$ & $5.63 \pm 9.58$ & $6.21 \pm 9.07$ & $5.22 \pm 11.22$ \\
\hline Parameter & Category & $\%$ ( $n$ absolute $)$ & $\%$ ( $n$ absolute $)$ & $\%(n$ absolute $)$ & $\%(n$ absolute $)$ \\
\hline \multirow[t]{4}{*}{ Body-mass-index $\left(\mathrm{kg} / \mathrm{m}^{2}\right)$} & $<18.5$ & $0.12(1)$ & $0.45(3)$ & $0.29(1)$ & $0.37(1)$ \\
\hline & 18.5 to $<25$ & $28.28(224)$ & $47.84(321)$ & $30.61(105)$ & $51.65(141)$ \\
\hline & 25 to $<30$ & $52.53(416)$ & $31.74(213)$ & $51.60(177)$ & $32.23(88)$ \\
\hline & $\geq 30$ & $19.07(151)$ & $19.82(133)$ & $17.49(60)$ & $15.75(43)$ \\
\hline Physical activity ${ }^{\mathrm{e}}$ & Active & $57.95(459)$ & $68.41(375)$ & 60.35 (207) & $60.07(164)$ \\
\hline
\end{tabular}

${ }^{a}$ Weight-stable subjects of KORA F4, whose weight gain or loss did not exceed more than $0.5 \%$ per year since their body weight was measured in KORA S4 (on average seven years prior F4 examination)

${ }^{b}$ Parameters derived from the BIA-measurements which were only conducted in KORA S4

${ }^{c}$ Fat free mass index

d Body fat mass index

e Physical Activity (active: $>1 \mathrm{~h}$ sport per week on a regular basis; inactive: $<1 \mathrm{~h}$ sport per week)

Also the mean FT4 value in the BMI category $<18.5$ consisting of 4 subjects was significantly higher as compared to the other BMI categories. No differences among categories were found for the TSH concentrations (Table 2).

After correction for multiple testing we obtained 154 statistically significant associations between various serum metabolite concentrations, metabolite ratios, and FT4. Significant associations between metabolites and TSH concentrations were entirely lacking in the main analysis as well as in the subanalyses. Selected statistically significant associations of FT4 with different metabolite concentrations for the KORA F4 population are shown in Table 3. Different parameters such as the $\beta$-estimate derived from the adjusted linear model are given, as well as the adjusted $p$ value, the adjusted $\mathrm{R}^{2}$ and the $\mathrm{p}$-gain. The full list of all obtained statistically significant associations can be found in Supplementary Table S2. The results of the subanalyses are given in the Supplementary Tables S3-S7.

\subsection{FT4 and serum acylcarnitines}

For a series of different serum acylcarnitines, statistically significant associations with serum FT4 concentrations were detected, and all of them showed a positive direction (of the $\beta$-estimate). This could be indicative of an overall elevated transport and subsequent $\beta$-oxidation of fatty acids with higher serum FT4 concentration. For acetylcarnitine (C2) as the quantitatively most important serum acylcarnitine the adjusted $p$ value was $4.46 \times 10^{-10}$. Similarly strong associations were detected for C16:1, C14:2, C18:2, the sums of all acylcarnitines, long-chain acylcarnitines, even-numbered acylcarnitines, and acylcarnitines carrying monounsaturated or polyunsaturated fatty acids. Special ratios, such as $\mathrm{C} 2$ by $\mathrm{C} 0$ or the sum of 
Table 2 Mean $( \pm \mathrm{SD})$ of FT4 hormone concentration (pmol/l) and TSH hormone concentration (mIU/L) across different strata of age, BMI $\left(\mathrm{kg} / \mathrm{m}^{2}\right)$, or physical activity for the KORA F4 participants $(n=1463)$, the weight-stable subset $(n=616)$ and the obese participants (BMI $\left.\geq 30 \mathrm{~kg} / \mathrm{m}^{2} ; n=284\right)$

\begin{tabular}{|c|c|c|c|c|c|c|c|c|c|c|}
\hline & & \multicolumn{3}{|c|}{ KORA F4 $(n=1463)$} & \multicolumn{3}{|c|}{ Weight-stable $^{\mathrm{a}}(n=616)$} & \multicolumn{3}{|c|}{ Obese subset $(n=284)$} \\
\hline & & FT4 & & TSH & FT4 & & TSH & FT4 & & TSH \\
\hline \multirow{7}{*}{$\begin{array}{l}\text { Parameter } \\
\text { Age (years) }\end{array}$} & Category & Mean \pm SD & & Mean \pm SD & Mean \pm SD & & Mean \pm SD & Mean \pm SD & & Mean \pm SD \\
\hline & $31-40$ & $13.34 \pm 1.59$ & & $1.54 \pm 0.67$ & $13.43 \pm 1.59$ & & $1.52 \pm 0.54$ & $13.00 \pm 1.54$ & & $1.44 \pm 0.66$ \\
\hline & $41-50$ & $13.23 \pm 1.61$ & & $1.47 \pm 0.67$ & $13.10 \pm 1.65$ & & $1.46 \pm 0.68$ & $13.35 \pm 1.65$ & & $1.54 \pm 0.58$ \\
\hline & $51-60$ & $13.41 \pm 1.54$ & & $1.40 \pm 0.70$ & $13.34 \pm 1.36$ & & $1.42 \pm 0.71$ & $13.34 \pm 1.45$ & & $1.48 \pm 0.61$ \\
\hline & $61-70$ & $13.88 \pm 1.68$ & $*$ & $1.41 \pm 0.64$ & $13.80 \pm 1.60$ & $*_{\text {of }} 2$ & $1.34 \pm 0.62$ & $13.93 \pm 1.71$ & & $1.58 \pm 0.68$ \\
\hline & $71-80$ & $14.45 \pm 1.72$ & $*$ & $1.33 \pm 0.70$ & $14.43 \pm 1.70$ & $\begin{array}{l}*_{\text {of }} \\
1-3\end{array}$ & $1.25 \pm 0.66$ & $14.49 \pm 1.85$ & 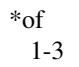 & $1.48 \pm 0.80$ \\
\hline & $\geq 81$ & $14.22 \pm 2.05$ & & $0.91 \pm 0.59$ & $14.22 \pm 2.05$ & & $0.91 \pm 0.59$ & $15.45 \pm 3.18$ & & $0.43 \pm 0.01$ \\
\hline \multirow{4}{*}{$\begin{array}{l}\text { BMI } \\
\left(\mathrm{kg} / \mathrm{m}^{2}\right)\end{array}$} & $<18.5$ & $16.65 \pm 1.04$ & $*$ & $1.23 \pm 0.59$ & $16.90 \pm 1.41$ & * & $1.23 \pm 0.89$ & & & \\
\hline & $\begin{array}{c}18.5 \text { to } \\
<25\end{array}$ & $13.50 \pm 1.62$ & & $1.46 \pm 0.73$ & $13.49 \pm 1.66$ & & $1.41 \pm 0.71$ & & & \\
\hline & 25 to $<30$ & $13.44 \pm 1.63$ & & $1.41 \pm 0.65$ & $13.49 \pm 1.50$ & & $1.36 \pm 0.59$ & & & \\
\hline & $\geq 30$ & $13.55 \pm 1.69$ & & $1.50 \pm 0.65$ & $13.46 \pm 1.73$ & & $1.54 \pm 0.69$ & & & \\
\hline \multirow{2}{*}{$\begin{array}{l}\text { Physical } \\
\text { activity }\end{array}$} & Active & $13.45 \pm 1.57$ & $\#$ & $1.44 \pm 0.69$ & $13.55 \pm 1.55$ & $\#$ & $1.38 \pm 0.65$ & $13.51 \pm 1.55$ & & $1.49 \pm 0.61$ \\
\hline & Inactive & $13.55 \pm 1.73$ & & $1.45 \pm 0.66$ & $13.41 \pm 1.71$ & & $1.46 \pm 0.67$ & $13.59 \pm 1.80$ & & $1.51 \pm 0.68$ \\
\hline
\end{tabular}

a Weight-stable subjects of KORA F4, whose weight gain or loss did not exceed more than $0.5 \%$ per year since their body weight was measured in KORA S4 (on average seven years prior $\mathrm{F} 4$ examination)

${ }^{\mathrm{b}}$ Physical Activity (active: $>1 \mathrm{~h}$ sport per week on a regular basis; inactive: less than $1 \mathrm{~h}$ sport per week)

* pairwise $t$ test was significant

\# levene test resulted in rejection of equal variances

$\mathrm{C} 2$ and $\mathrm{C} 3$ by $\mathrm{C} 0$ were suggested as valid measures of $\beta$-oxidation of fatty acids (Pande 1975; Roschinger et al. 2000) and they were both positively associated with FT4 hormone concentrations in our study (Table 3, Fig. 2a).

\subsection{FT4 and serum phosphatidylcholines}

The group of phosphatidylcholines consists of different PC diacyl (aa), PC acyl-alkyl (ae), and lysoPC acyl (a) compounds. Numerous associations between FT4 and PCs or PC ratios were observed. Generally, an increase in FT4 levels was associated with lower concentrations of PCs. This was reflected by the sum of all PCs, and the different sums of PCs containing saturated, monounsaturated, polyunsaturated, or short-chain fatty acids (Fig. 2b). These results are in line with the associations found for the sums of PC aa, PC ae, or lysoPC with FT4 concentrations. Furthermore, single PCs such as PC aa C34:4, PC aa 36:1, PC ae C40:1, PC ae C42:2, lysoPC a C14:0, or lysoPC a C18:1 were all inversely associated with increasing serum FT4 concentrations. Regarding metabolite ratios, we received some indication for an increase in saturation and chain length for $\mathrm{PC}$ aa by $\mathrm{PC}$ aa ratios, such as $\mathrm{PC}$ aa C36:6 by $\mathrm{PC}$ aa $\mathrm{C} 42: 0, \mathrm{PC}$ aa $\mathrm{C} 36: 5$ by $\mathrm{PC}$ aa $\mathrm{C} 42: 0$ or PC aa 36:0 by PC aa C38:0 with elevated FT4 levels. For PC ae by $\mathrm{PC}$ ae ratios, an increase in desaturation and chain- length with higher FT4 concentrations was observed, e.g., $\mathrm{PC}$ ae $\mathrm{C} 42: 2$ by PC ae $\mathrm{C} 42.5$, PC ae C40:1 by PC ae C40:5 or PC ae C38:2 by PC ae C42:5.

\subsection{Subanalyses}

\subsubsection{Weight-stable subsample}

Restricting our sample to the weight-stable subset and adjusting for FFMI instead of BMI resulted in a substantial loss of significant associations with FT4 as compared to the results in the entire sample (Supplementary Table S3). Although $\beta$-estimates are similar in both analyses, statistical power was lower due to the smaller size of the weight-stable group. Still, long-chain acylcarnitines, the sums of longchain acylcarnitines and of monounsaturated acylcarnitines were significantly positively associated with FT4 levels in this subgroup. The significant associations between single PC concentrations and FT4 were similar to the results of the entire sample. However, significant associations with PC ratios are largely missing, indicating loss of statistical power as a consequence of the reduced sample size. Adjusting the linear models for BMI instead of FFMI in the weight-stable subgroup resulted in almost the same significant associations with FT4, suggesting no distinct effect of the more precise adjustment for lean body mass. 
Table 3 Selected metabolic traits significantly associated with FT4 in a linear regression model adjusted for age, sex, $\mathrm{BMI}$, and batch in the KORA F4 sample
The full results are given in the Supplementary Table S2

$A C$ acylcarnitines, even even numbered, long long chain, med. medium chain, short short chain, sat. saturated, unsat. unsaturated

${ }^{a}$ For multiple testing adjusted $p$ value

b Adjusted $\mathrm{R}^{2}$ of the linear model

c p-gain, fold decrease in the $p$ value association for the pair of metabolites, compared to the lowest of two $p$ values for the single metabolites

\begin{tabular}{|c|c|c|c|c|c|c|}
\hline Trait & $\begin{array}{l}\text { Mean } \\
(\mu \mathrm{mol} / 1)\end{array}$ & SD & Beta & Adj. $P$ value ${ }^{\mathrm{a}}$ & $\mathrm{R}^{2}$ adj $^{\mathrm{b}}$ & P-Gain \\
\hline \multicolumn{7}{|l|}{ Acylcarnitines } \\
\hline$\Sigma \mathrm{AC}$ & 10.81 & 3.24 & 0.11 & $1.23 \times 10^{-10}$ & 0.14 & \\
\hline$\Sigma$ long AC & 0.40 & 0.09 & 0.10 & $1.25 \times 10^{-10}$ & 0.21 & \\
\hline$\Sigma$ even AC & 9.97 & 3.16 & 0.11 & $1.29 \times 10^{-10}$ & 0.14 & \\
\hline$\Sigma$ poly AC & 8.32 & 2.85 & 0.11 & $2.41 \times 10^{-10}$ & 0.13 & \\
\hline$\Sigma$ mono $\mathrm{AC}$ & 1.73 & 0.46 & 0.10 & $2.12 \times 10^{-08}$ & 0.16 & \\
\hline$\Sigma$ med. AC & 1.12 & 0.36 & 0.09 & $3.00 \times 10^{-06}$ & 0.14 & \\
\hline C18:1 & 0.13 & 0.04 & 0.12 & $7.04 \times 10^{-14}$ & 0.18 & \\
\hline C16:1 & 0.04 & 0.01 & 0.11 & $6.15 \times 10^{-11}$ & 0.17 & \\
\hline $\mathrm{C} 14: 2$ & 0.03 & 0.01 & 0.11 & $3.17 \times 10^{-10}$ & 0.14 & \\
\hline $\mathrm{C} 2$ & 7.85 & 2.77 & 0.11 & $4.46 \times 10^{-10}$ & 0.13 & \\
\hline $\mathrm{C} 18: 2$ & 0.05 & 0.01 & 0.09 & $6.44 \times 10^{-07}$ & 0.18 & \\
\hline $\mathrm{C} 12: 1$ & 0.14 & 0.05 & 0.09 & $1.32 \times 10^{-06}$ & 0.11 & \\
\hline $\mathrm{C} 12$ & 0.13 & 0.05 & 0.09 & $1.81 \times 10^{-06}$ & 0.12 & \\
\hline $\mathrm{C} 10$ & 0.34 & 0.16 & 0.08 & $3.66 \times 10^{-05}$ & 0.09 & \\
\hline C10:1 & 0.16 & 0.06 & 0.07 & $2.37 \times 10^{-04}$ & 0.13 & \\
\hline $\mathrm{C} 14: 1$ & 0.14 & 0.05 & 0.06 & $1.69 \times 10^{-02}$ & 0.25 & \\
\hline $\mathrm{C} 8$ & 0.21 & 0.09 & 0.06 & $2.37 \times 10^{-02}$ & 0.10 & \\
\hline$(\mathrm{C} 2+\mathrm{C} 3) / \mathrm{C} 0$ & 0.24 & 0.08 & 0.11 & $1.72 \times 10^{-10}$ & 0.14 & \\
\hline $\mathrm{C} 2 / \mathrm{C} 0$ & 0.23 & 0.08 & 0.11 & $3.12 \times 10^{-10}$ & 0.15 & \\
\hline$\Sigma$ PCs & 1984.61 & 355.11 & -0.11 & $3.55 \times 10^{-11}$ & 0.14 & \\
\hline$\Sigma$ mono PCs & 348.02 & 86.20 & -0.11 & $8.18 \times 10^{-11}$ & 0.07 & \\
\hline$\Sigma$ poly PCs & 1592.39 & 279.37 & -0.11 & $9.87 \times 10^{-10}$ & 0.15 & \\
\hline$\Sigma$ sat. PCs & 44.21 & 7.90 & -0.09 & $2.61 \times 10^{-06}$ & 0.12 & \\
\hline$\Sigma$ short PCs & 759.09 & 161.58 & -0.11 & $3.37 \times 10^{-11}$ & 0.25 & \\
\hline$\Sigma \mathrm{PC}$ aa & 1786.89 & 333.22 & -0.12 & $8.01 \times 10^{-12}$ & 0.14 & \\
\hline$\Sigma$ short $\mathrm{PC}$ aa & 720.81 & 156.95 & -0.11 & $2.82 \times 10^{-11}$ & 0.25 & \\
\hline$\Sigma$ mono PC aa & 323.42 & 83.04 & -0.11 & $9.75 \times 10^{-11}$ & 0.07 & \\
\hline$\Sigma$ poly $\mathrm{PC}$ aa & 1435.77 & 261.53 & -0.11 & $2.28 \times 10^{-10}$ & 0.15 & \\
\hline$\Sigma$ sat. PC aa & 27.71 & 5.60 & -0.09 & $9.30 \times 10^{-06}$ & 0.11 & \\
\hline$\Sigma$ long PC aa & 47.11 & 12.34 & -0.06 & $1.08 \times 10^{-02}$ & 0.07 & \\
\hline $\mathrm{PC}$ aa $\mathrm{C} 34: 4$ & 2.26 & 0.83 & -0.14 & $2.02 \times 10^{-16}$ & 0.07 & \\
\hline PC aa C36:1 & 53.43 & 13.58 & -0.13 & $7.41 \times 10^{-15}$ & 0.10 & \\
\hline $\mathrm{PC}$ aa $\mathrm{C} 36: 6 / \mathrm{PC}$ aa $\mathrm{C} 42: 0$ & 1.95 & 0.80 & -0.14 & $7.46 \times 10^{-16}$ & 0.06 & $7.90 \times 10^{+05}$ \\
\hline $\mathrm{PC}$ aa $\mathrm{C} 36: 5 / \mathrm{PC}$ aa $\mathrm{C} 42: 0$ & 51.17 & 24.93 & -0.13 & $2.97 \times 10^{-14}$ & 0.10 & $5.84 \times 10^{+04}$ \\
\hline $\mathrm{PC}$ aa $\mathrm{C} 36: 0 / \mathrm{PC}$ aa $\mathrm{C} 38: 0$ & 0.83 & 0.13 & -0.11 & $3.23 \times 10^{-09}$ & 0.04 & $1.34 \times 10^{+08}$ \\
\hline$\Sigma$ sat. PC ae & 16.50 & 2.81 & -0.08 & $7.06 \times 10^{-05}$ & 0.12 & \\
\hline$\Sigma$ mono PC ae & 24.60 & 4.98 & -0.07 & $6.06 \times 10^{-04}$ & 0.17 & \\
\hline$\Sigma$ short PC ae & 38.28 & 8.23 & -0.06 & $1.03 \times 10^{-02}$ & 0.19 & \\
\hline $\mathrm{PC}$ ae $\mathrm{C} 40: 1$ & 1.69 & 0.40 & -0.11 & $2.11 \times 10^{-10}$ & 0.08 & \\
\hline $\mathrm{PC}$ ae $\mathrm{C} 42: 2$ & 0.68 & 0.15 & -0.09 & $4.58 \times 10^{-07}$ & 0.12 & \\
\hline $\mathrm{PC}$ ae $\mathrm{C} 42: 2 / \mathrm{PC}$ ae $\mathrm{C} 42: 5$ & 0.29 & 0.06 & -0.15 & $9.27 \times 10^{-20}$ & 0.07 & $4.94 \times 10^{+12}$ \\
\hline $\mathrm{PC}$ ae $\mathrm{C} 40: 1 / \mathrm{PC}$ ae $\mathrm{C} 40: 5$ & 0.48 & 0.10 & -0.14 & $2.92 \times 10^{-17}$ & 0.09 & $7.22 \times 10^{+06}$ \\
\hline $\mathrm{PC}$ ae $\mathrm{C} 38: 2 / \mathrm{PC}$ ae $\mathrm{C} 42: 5$ & 0.92 & 0.22 & -0.14 & $2.97 \times 10^{-16}$ & 0.08 & $9.92 \times 10^{+06}$ \\
\hline$\Sigma$ unsat. lysoPCs & 63.67 & 17.36 & -0.08 & $2.92 \times 10^{-05}$ & 0.24 & \\
\hline$\Sigma$ lysoPCs & 191.45 & 40.77 & -0.08 & $6.32 \times 10^{-05}$ & 0.19 & \\
\hline$\Sigma$ sat. lysoPCs & 127.78 & 26.46 & -0.07 & $2.02 \times 10^{-03}$ & 0.14 & \\
\hline lysoPC a C14:0 & 3.23 & 0.84 & -0.09 & $1.37 \times 10^{-06}$ & 0.05 & \\
\hline lysoPC a C18:1 & 19.77 & 5.79 & -0.08 & $3.40 \times 10^{-05}$ & 0.22 & \\
\hline
\end{tabular}




\subsubsection{Males versus females}

The acylcarnitine results for men were very much comparable to the results in the whole sample while the results for the female participants varied slightly (Supplementary Table S4). Here, the positive associations with the sums of acylcarnitines were still present, but regarding the single concentrations, only C18:1, C14:2, C18:2, C2, and C16:1 are positively associated with elevated FT4 hormone concentrations. Testing for differences of the estimated coefficients between men and women revealed no statistical significance. The associations between PCs and FT4 levels in men and women were in line with the results for the entire study population.

\subsubsection{Obese versus non-obese}

The associations with FT4 levels found for the whole population and the non-obese participants were similar for both, the acylcarnitine and the PC concentrations (see Supplementary Table S5). In the obese group, many significant associations identified in the entire group were lacking which is likely due to sample size reasons. This is supported by the results of the $\beta$-estimates which were largely comparable in size and direction to those obtained in the entire group or the non-obese participants, although not reaching statistical significance (data not shown). Still, significant associations were identified for long-chain acylcarnitines with FT4 in the group of obese participants.

\subsubsection{Physical activity}

The acylcarnitine results for physically inactive participants paralleled the results in the whole sample, showing an enhanced oxidation activity with no preference of acylcarnitine type. The results for the active participants varied slightly (Supplementary Table S6). Here, the positive associations with most sums of acylcarnitines were also present, but regarding individual compounds, only C18:1, C14:2, C16:1 and C2 were still positively associated with elevated FT4 hormone concentrations. Testing for differences of the estimated coefficients between groups revealed no statistical significance. The associations between PCs and FT4 levels in active and inactive participants were in line with the results for the entire study population. For the inactive participants, however, most associations regarding metabolite ratios were missing.

\subsubsection{Age groups $(\leq 60$ years versus $>60$ years)}

The results for the $\leq 60$ age group $(n=1132)$ are very similar to those of the entire study population concerning significance and estimate size. In the $>60$ age group, associations with most sums of acylcarnitines and sums of PCs were still present. However on the individual compound level and concerning PC ratios several significant associations with FT4 present in the entire population and the younger age group were lacking. This could be due to sample size reasons ( $>60$ years; $n=331$ ). However, testing for differences of the estimated coefficients between age groups revealed no statistical significance. Results are shown in Supplementary Table S7.

\subsubsection{Influence of insulin, liver, kidney function, or inflammation parameters}

Almost all identified associations between FT4 and different serum metabolites were independent of serum insulin, and parameters of liver and kidney function, or inflammatory biomarkers such as glutamate pyruvate transaminase, glutamic oxaloacetic transaminase, gammaglutamyl transpeptidase, cystatin C, creatinine, C-reactive protein, or white blood cell count. Only the associations between FT4 and C18:1, PC aa C38:5, PC aa C40:4, PC aa C40:5, PC aa C42:6, PC ae C40:0, PC ae C42:1 and lysoPC a $\mathrm{C} 16: 1$ were influenced by gamma-glutamyl transpeptidase, and the association between FT4 and lysoPC a C18:2 was affected by $\mathrm{C}$-reactive protein.

\section{Discussion}

In this euthyroid population, after overnight fasting, strong associations between serum FT4 and serum metabolite concentrations were found. With higher FT4 levels, the concentration of acylcarnitines as well as the ratio of $\mathrm{C} 2$ by $\mathrm{C} 0$ increased, indicating an overall increase of $\beta$-oxidation of fatty acids. In addition, the inverse associations between FT4 and PC metabolites are reflective of a uniform decline in serum PC concentrations. Figure 3 illustrates a schematic description of cellular fatty acid transport, $\beta$-oxidation, and how these are linked to our found associations between FT4, acylcarnitines, and PCs. These results were supported by the associations found in stratified analyses and strengthened by their independency of serum insulin and different liver, kidney function, and inflammation parameters. In euthyroid adults, TSH concentrations were not associated with serum metabolites. Also, no effect of FT4 on serum amino acids concentrations was found.

Knowledge of how thyroid hormones exert their effects has further increased since the successful molecular cloning of nuclear thyroid hormone receptors in the mid 90's (Flamant et al. 2007; Ribeiro et al. 1998; Wagner et al. 1995; Yen 2001). Most of the physiological effects of thyroid hormones are exerted through thyroid hormone 

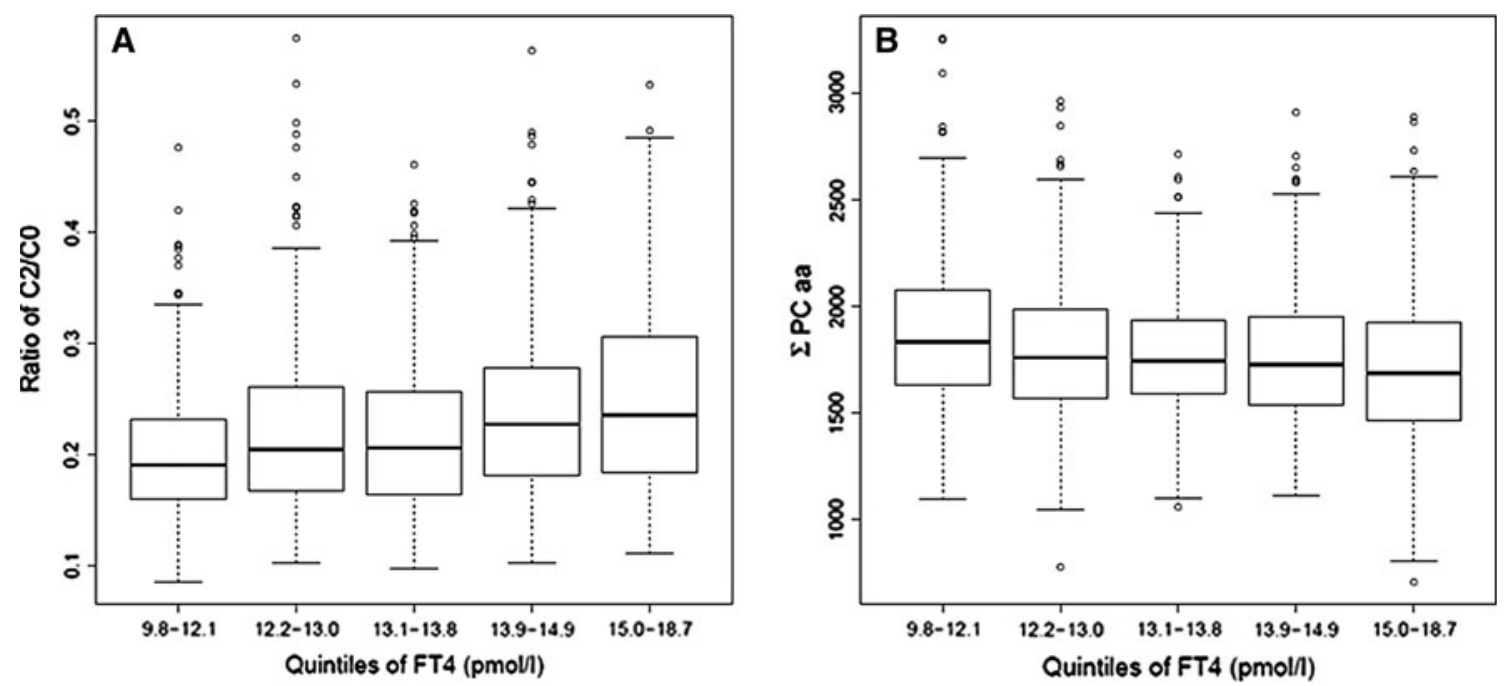

Fig. 2 Boxplot of serum C2/C0 (acetylcarnitine/free carnitine) concentrations (a) and the sum of PC aa concentrations (b) by quintiles of FT4 (pmol/L), for the KORA F4 population

receptor complexes and the response elements of regulatory regions of target genes (Biondi 2012a; Brix et al. 2011). Nevertheless, not all thyroid hormone effects are attributable to nuclear mediated pathways (Moreno et al. 2008) and an increasing number of non-genetic effects such as the transportation of ions, e.g., calcium, sodium, and potassium across plasma membranes or glucose and amino acids have been described lately (Biondi et al. 2002; Fazio et al. 2004). From a clinical point of view, it is clear that minor changes in thyroid hormone concentrations cause distinct metabolic effects (Kim 2008). In a recent microarray study by Clement et al. (2002) on the transcriptome of the vastus lateralis, a daily dosage of $75 \mu \mathrm{g}$ of T4 was administered to healthy male volunteers with the result of 22 genes involved in mitochondrial energy metabolism being up-regulated. At the cellular level, T4 is converted to T3, and T3 is considered the active hormone. It is evident that intracellular $\mathrm{T} 3$ concentrations do not necessarily correspond to blood T3 concentrations. Although serum FT3 concentrations were measured in our study as well, the applied analytic method did not fulfil the strict quality control criteria in the end (Thienpont et al. 2010). Thus, FT3 data could not be used in this project. However, our results and the findings of the microarray study argue for an independent effect of FT4 on the mitochondrial level. There is no indication of an association between serum TSH concentration and serum metabolites. It is serum FT3 and FT4 that reveal biological effects at the cellular level. In an euthyroid situation, the serum TSH concentration was not strongly linked to the concentration of FT4 (correlation coefficient $r=-0.15$ ) in our study.

In a human experimental setting, it has recently been described that all plasma acylcarnitines (except for C3, C4, and C5) showed a strong increase in times of elevated $\beta$-oxidation, e.g., during fasting and or exercise (Krug et al. 2012). Concomitantly, plasma free carnitine (C0) decreases. In this project blood samples from all participants were drawn in fasting state, i.e., after overnight fast (minimum of $8 \mathrm{~h}$ ). Thus, all study participants were in a catabolic metabolic state which is characterised by low plasma insulin concentrations, increased plasma concentrations of non-esterified fatty acids and ketone bodies, and an enhanced gluconeogenesis. Under this fasting condition, our results strongly indicate elevated $\beta$-oxidation with increasing plasma FT4 concentrations. Results from single acylcarnitine concentrations, sums of different acylcarnitine groups, as well as the ratio of C2 by C0 (Pande 1975; Roschinger et al. 2000) support this conclusion. The observed decreasing serum PC concentrations with higher FT4 levels are probably the consequence of this enhanced lipid oxidation, and therefore underline our hypothesis.

Krug et al. (2012) further demonstrated that plasma concentrations of specific amino acids, especially branched-chain amino acids, increased during fasting status, indicating enhanced gluconeogenesis. Although, a strong relationship between branched-chain amino acid serum concentrations and fat free mass in fasted state has been described for the present population (Jourdan et al. 2012), we did not observe significant associations between serum amino acid concentrations and FT4 levels in this project. Thus, we conclude that FT4 does not affect gluconeogenesis in euthyroid and fasting subjects. This finding affirms the notion that we do not simply see the effects of fasting status in our study but a specific effect attributable to the thyroid hormone FT4 which is characterised by enhanced transport of fatty acids in form of carnitine acylesters to the mitochondrion and subsequent $\beta$-oxidation. To the best of 


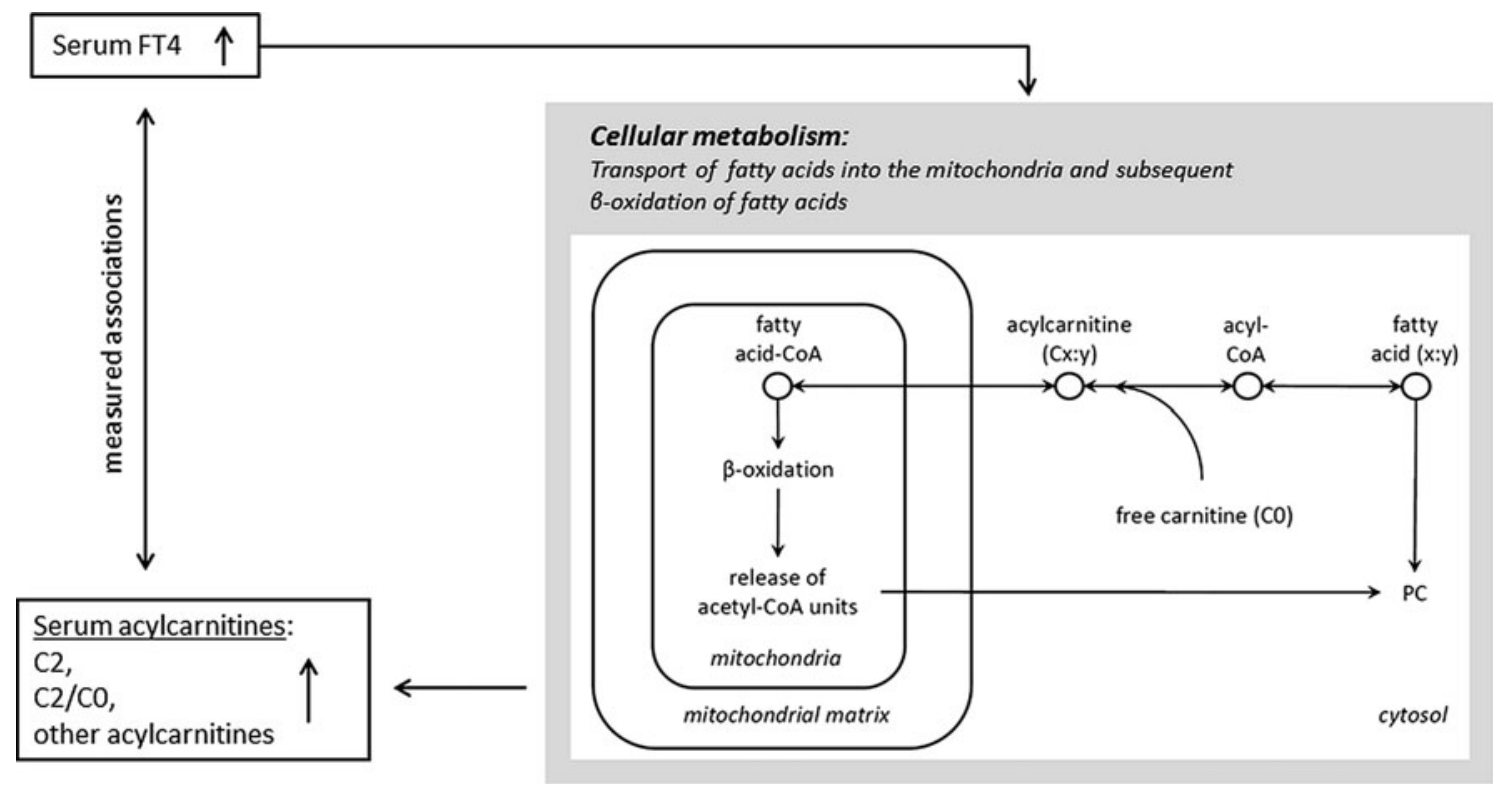

Fig. 3 Schematic description of the cellular fatty acid transport and $\beta$-oxidation as the metabolic basis for the observed associations between FT4 and serum acylcarnitines and phosphatidylcholines (PCs)

our knowledge no study directly comparable to ours has been performed so far. Most recent studies using transcriptomics or proteomics techniques investigated effects of $\mathrm{T} 3$ administration. In these studies effects of $\mathrm{T} 3$ on proteins of gluconeogenic pathways have been described; a review is given by Silvestri et al. (2011).

For treatment of obesity, $\mathrm{T} 3$ and $\mathrm{T} 4$ in varying doses and duration have been administered to euthyroid obese subjects during caloric deprivation to enhance weight loss and a systematic review on this issue is given by Kaptein et al. (2009). The authors summarised that available studies are insufficient to draw conclusions on beneficial or adverse effects of T3 or T4 administration; however, because of possible side-effect they do not advise on treatment of obesity with T4 or T3 (Kaptein et al. 2009). The results of the subanalyses presented here did not suggest specific differences by fat free mass (analysis in weight-stable subjects) or between obese and non-obese subjects. Also, subanalyses by sex, age, and physical activity provided largely similar results across subgroups. Thus, we conclude that the positive association between FT4 and $\beta$-oxidation of fatty acids after overnight fast is not dependent on lean body mass, body fat mass, age, sex, or habitual physical activity. In other words, we gained no indication for obesity being a consequence of altered fatty acid oxidation in fasting status as related to serum FT4 concentrations in euthyroid subjects. Further, no impact of serum insulin, or biomarkers of liver or kidney function or inflammation on the associations between FT4 and AC were observed. This supports the stability of our results and argues for an independent effect of FT4. However, this lack of impact could also be due to the characteristics of our study population. The selection of our participants was accompanied by an exclusion of elderly participants which might represent a risk group, e.g., for inflammation.

\section{Strength and limitations}

The human serum metabolome is currently characterised in many studies using different analytic approaches (Psychogios et al. 2011), including the method applied here. To the best of our knowledge, the association of FT4, TSH, and serum metabolites was not explored before in an euthyroid population. The participants of our study represent a random sample of the underlying population. In such a population with a heterogeneous metabolic makeup influenced by different environmental factors, genetic predispositions, and lifestyles, the interpretation of metabolic profiling is quite ambitious (Suhre et al. 2010). The metabolite concentrations which are reported in the present paper were identified by means of the Absolute $I D Q^{\mathrm{TM}}$ p150 kit. This technology allows the automated quantification of many metabolites with a focus on amino acids, acylcarnitines, and PCs for many samples at a time (Altmaier et al. 2011; Wenk 2005). Thus, the kit is well suitable to assess a suggested impact of thyroid hormones on energy metabolism, especially lipid metabolism. The BIA measurements did only take place in KORA S4. For the analyses of associations with FT4 and the metabolite concentrations in the linear models with the FFMI instead of the BMI as adjustment variable only the results of the 
BIA measurements at baseline examination (KORA S4) were available. Assuming that the participants' body composition is fairly stable in weight-stable subjects, those analyses were restricted to the weight-stable subjects in order to account for this.

\section{Conclusion}

Our results indicate that in a euthyroid population after overnight fast, serum FT4 concentrations are strongly linked to serum acylcarnitines and PCs, indicating enhanced transport of fatty acids to the mitochondrion and subsequent $\beta$-oxidation. These findings were stable as they could be largely reproduced in different subsets of the population.

Acknowledgments The KORA study group consists of A. Peters (speaker), J. Heinrich, R. Holle, R. Leidl, C. Meisinger, K. Strauch and their coworkers, who are responsible for the design and conduct of the KORA studies. We gratefully acknowledge the contribution of all members of field staffs who were involved in planning and conducting the MONICA/KORA Augsburg studies. Finally, we express our appreciation to all study participants for donating their blood and time. We thank Dr. Werner Römisch-Margl, Julia Scarpa, and Katharina Sckell for metabolomics measurements performed at the Helmholtz Zentrum München, Genome Analysis Centre, Metabolomics Core Facility. This study was supported by a Grant from the German Federal Ministry of Education and Research (BMBF) to the German Center for Diabetes Research (DZD e.V.).

Funding The KORA research platform (KORA, Cooperative Research in the Region of Augsburg) was initiated and financed by the Helmholtz Zentrum München-German Research Centre for Environmental Health, which is funded by the German Federal Ministry of Education and Research and by the State of Bavaria. Furthermore, KORA research was supported within the Munich Center of Health Sciences (MC Health), Ludwig-Maximilians-Universität, as part of LMUinnovativ. This study was further supported by the German Research Foundation (DFG Vo 955/10-2).

\section{References}

Altmaier, E., Kastenmuller, G., Romisch-Margl, W., Thorand, B., Weinberger, K. M., Adamski, J., et al. (2009). Variation in the human lipidome associated with coffee consumption as revealed by quantitative targeted metabolomics. Molecular Nutrition \& Food Research, 53(11), 1357-1365.

Altmaier, E., Kastenmuller, G., Romisch-Margl, W., Thorand, B., Weinberger, K. M., Illig, T., et al. (2011). Questionnaire-based self-reported nutrition habits associate with serum metabolism as revealed by quantitative targeted metabolomics. European Journal of Epidemiology, 26(2), 145-156.

Altmaier, E., Ramsay, S. L., Graber, A., Mewes, H. W., Weinberger, K. M., \& Suhre, K. (2008). Bioinformatics analysis of targeted metabolomics - uncovering old and new tales of diabetic mice under medication. Endocrinology, 149(7), 3478-3489.
Assfalg, M., Bertini, I., Colangiuli, D., Luchinat, C., Schafer, H., Schutz, B., et al. (2008). Evidence of different metabolic phenotypes in humans. Proceedings of the National Academy of Sciences of the United States of America, 105(5), 1420-1424.

Biondi, B. (2012a). Mechanisms in endocrinology: heart failure and thyroid dysfunction. European Journal of Endocrinology, 167(5), 609-618.

Biondi, B. (2012b). Natural history, diagnosis and management of subclinical thyroid dysfunction. Best Practice and Research Clinical Endocrinology and Metabolism, 26(4), 431-446.

Biondi, B., Palmieri, E. A., Lombardi, G., \& Fazio, S. (2002). Subclinical hypothyroidism and cardiac function. Thyroid, 12(6), 505-510.

Boelaert, K., \& Franklyn, J. A. (2005). Thyroid hormone in health and disease. Journal of Endocrinology, 187(1), 1-15.

Brabant, G., Beck-Peccoz, P., Jarzab, B., Laurberg, P., Orgiazzi, J., Szabolcs, I., et al. (2006). Is there a need to redefine the upper normal limit of TSH? European Journal of Endocrinology, 154(5), 633-637.

Brix, K., Fuhrer, D., \& Biebermann, H. (2011). Molecules important for thyroid hormone synthesis and action-known facts and future perspectives. Thyroid Research, 4(Suppl 1), S9.

Clement, K., Viguerie, N., Diehn, M., Alizadeh, A., Barbe, P., Thalamas, C., et al. (2002). In vivo regulation of human skeletal muscle gene expression by thyroid hormone. Genome Research, 12(2), 281-291.

Demers, L. M., \& Spencer, C. A. (2003). Laboratory Support for the diagnosis and monitoring of thyroid disease. National Academy of Clinical Biochemistry Laboratory Medicine Practice Guidelines. Washington: National Academy of Clinical Biochemistry.

Dumas, M. E., Wilder, S. P., Bihoreau, M. T., Barton, R. H., Fearnside, J. F., Argoud, K., et al. (2007). Direct quantitative trait locus mapping of mammalian metabolic phenotypes in diabetic and normoglycemic rat models. Nature Genetics, 39(5), 666-672.

Fazio, S., Palmieri, E. A., Lombardi, G., \& Biondi, B. (2004). Effects of thyroid hormone on the cardiovascular system. Recent Progress in Hormone Research, 59, 31-50.

Flamant, F., Gauthier, K., \& Samarut, J. (2007). Thyroid hormones signaling is getting more complex: STORMs are coming. Molecular Endocrinology, 21(2), 321-333.

Goek, O. N., Doring, A., Gieger, C., Heier, M., Koenig, W., Prehn, C., et al. (2012). Serum metabolite concentrations and decreased GFR in the general population. American Journal of Kidney Diseases, 60(2), 197-206.

Holle, R., Happich, M., Lowel, H., \& Wichmann, H. E. (2005). KORA-a research platform for population based health research. Gesundheitswesen, 67(Suppl 1), S19-S25.

Illig, T., Gieger, C., Zhai, G., Romisch-Margl, W., Wang-Sattler, R., Prehn, C., et al. (2010). A genome-wide perspective of genetic variation in human metabolism. Nature Genetics, 42(2), 137-141.

Jourdan, C., Petersen, A. K., Gieger, C., Doring, A., Illig, T., WangSattler, R., et al. (2012). Body fat free mass is associated with the serum metabolite profile in a population-based study. PLoS One, 7(6), e40009.

Kaptein, E. M., Beale, E., \& Chan, L. S. (2009). Thyroid hormone therapy for obesity and nonthyroidal illnesses: a systematic review. Journal of Clinical Endocrinology and Metabolism, 94(10), 3663-3675.

Kim, B. (2008). Thyroid hormone as a determinant of energy expenditure and the basal metabolic rate. Thyroid, 18(2), $141-144$.

Knudsen, N., Jorgensen, T., Rasmussen, S., Christiansen, E., \& Perrild, H. (1999). The prevalence of thyroid dysfunction in a 
population with borderline iodine deficiency. Clinical Endocrinology (Oxf), 51(3), 361-367.

Krug, S., Kastenmuller, G., Stuckler, F., Rist, M. J., Skurk, T., Sailer, M., et al. (2012). The dynamic range of the human metabolome revealed by challenges. FASEB Journal, 26(6), 2607-2619.

Kyle, U. G., Genton, L., Karsegard, L., Slosman, D. O., \& Pichard, C. (2001). Single prediction equation for bioelectrical impedance analysis in adults aged 20-94 years. Nutrition, 17(3), 248-253.

Meisinger, C., Lowel, H., Heier, M., Kandler, U., \& Doring, A. (2007). Association of sports activities in leisure time and incident myocardial infarction in middle-aged men and women from the general population: the MONICA/KORA Augsburg cohort study. European Journal of Cardiovascular Prevention and Rehabilitation, 14(6), 788-792.

Moreno, M., de Lange, P., Lombardi, A., Silvestri, E., Lanni, A., \& Goglia, F. (2008). Metabolic effects of thyroid hormone derivatives. Thyroid, 18(2), 239-253.

Nimptsch, K., Berg-Beckhoff, G., \& Linseisen, J. (2010). Effect of dietary fatty acid intake on prospective weight change in the Heidelberg cohort of the European prospective investigation into cancer and nutrition. Public Health Nutrition, 13(10), $1636-1646$.

Pande, S. V. (1975). A mitochondrial carnitine acylcarnitine translocase system. Proceedings of the National Academy of Sciences of the United States of America, 72(3), 883-887.

Panicker, V., Wilson, S. G., Walsh, J. P., Richards, J. B., Brown, S. J., Beilby, J. P., et al. (2010). A locus on chromosome 1p36 is associated with thyrotropin and thyroid function as identified by genome-wide association study. American Journal of Human Genetics, 87(3), 430-435.

Paternoster, R., Brame, R., Mazerolle, P., \& Piquero, A. (1998). Using the correct statistical test for the equality of regression coefficients. Criminology, 36(4), 859-866.

Psychogios, N., Hau, D. D., Peng, J., Guo, A. C., Mandal, R., Bouatra, S., et al. (2011). The human serum metabolome. PLoS One, 6(2), e16957.

Ribeiro, R. C., Apriletti, J. W., Wagner, R. L., West, B. L., Feng, W., Huber, R., et al. (1998). Mechanisms of thyroid hormone action: insights from X-ray crystallographic and functional studies. Recent Progress in Hormone Research, 53, 351-392. discussion 392-354.

Römisch-Margl, W., Prehn, C., Bogumil, R., Röhring, C., Suhre, K., \& Adamski, J. (2011). Procedure for tissue sample preparation and metabolite extraction for high-throughput targeted metabolomics. Metabolomics, 1-10.

Roschinger, W., Muntau, A. C., Duran, M., Dorland, L., IJIst, L., Wanders, R. J., et al. (2000). Carnitine-acylcarnitine translocase deficiency: metabolic consequences of an impaired mitochondrial carnitine cycle. Clinica Chimica Acta, 298(1-2), 55-68.

Ruckert, I. M., Heier, M., Rathmann, W., Baumeister, S. E., Doring, A., \& Meisinger, C. (2011). Association between markers of fatty liver disease and impaired glucose regulation in men and women from the general population: the KORA-F4-study. PLoS One, 6(8), e22932.

Seissler, J., Feghelm, N., Then, C., Meisinger, C., Herder, C., Koenig, W., et al. (2012). Vasoregulatory peptides pro-endothelin-1 and pro-adrenomedullin are associated with metabolic syndrome in the population-based KORA F4 study. European Journal of Endocrinology, 167(6), 847-853.

Silvestri, E., Lombardi, A., de Lange, P., Glinni, D., Senese, R., Cioffi, F., et al. (2011). Studies of complex biological systems with applications to molecular medicine: the need to integrate transcriptomic and proteomic approaches. Journal of Biomedicine and Biotechnology, 2011, 810242.

Suhre, K., Meisinger, C., Doring, A., Altmaier, E., Belcredi, P., Gieger, C., et al. (2010). Metabolic footprint of diabetes: a multiplatform metabolomics study in an epidemiological setting. PLoS One, 5(11), e13953.

Surks, M. I., Ortiz, E., Daniels, G. H., Sawin, C. T., Col, N. F., Cobin, R. H., et al. (2004). Subclinical thyroid disease: scientific review and guidelines for diagnosis and management. JAMA, 291(2), 228-238.

Thienpont, L. M., Van Uytfanghe, K., Beastall, G., Faix, J. D., Ieiri, T., Miller, W. G., et al. (2010). Report of the IFCC working group for standardization of thyroid function tests; part 2: free thyroxine and free triiodothyronine. Clinical Chemistry, 56(6), 912-920.

U.S. Department of Health and Human Services, Food and Drug Administration (FDA), Center for Drug Evaluation and Research (CDER), \& Center for Veterinary Medicine (CVM) (May 2001). Guidance for Industry - Bioanalytical Method Validation.

van Buuren, S., \& Groothuis-Oudshoorn, K. (2011). Mice: multivariate imputation by chained equations in R. Journal of Statistical Software, 45(3), 1-67.

Vinayavekhin, N., \& Saghatelian, A. (2009). Regulation of alkyldihydrothiazole-carboxylates (ATCs) by iron and the pyochelin gene cluster in Pseudomonas aeruginosa. ACS Chemical Biology, 4(8), 617-623.

Wagner, R. L., Apriletti, J. W., McGrath, M. E., West, B. L., Baxter, J. D., \& Fletterick, R. J. (1995). A structural role for hormone in the thyroid hormone receptor. Nature, 378(6558), 690-697.

Watson, A. D. (2006). Thematic review series: systems biology approaches to metabolic and cardiovascular disorders. Lipidomics: a global approach to lipid analysis in biological systems. Journal of Lipid Research, 47(10), 2101-2111.

Wenk, M. R. (2005). The emerging field of lipidomics. Nature Reviews Drug Discovery, 7, 594-610.

WHO. (2000). Obesity: preventing and managing the global epidemic. Report of a WHO consultation. Technical Report Series, $894,1-253$.

Yen, P. M. (2001). Physiological and molecular basis of thyroid hormone action. Physiological Reviews, 81(3), 1097-1142. 